\title{
Determinantes da retenção de peso pós-parto segundo a cor da pele em mulheres do Rio de Janeiro, Brasil
}

\author{
Ivyna Spinola Caetano Jordão e Gilberto Kac ${ }^{1,2}$
}

Como citar Jordão ISC, Kac G. Determinantes da retenção de peso pós-parto segundo a cor da pele em mulheres do Rio de Janeiro, Brasil. Rev Panam Salud Publica. 2005;18(6):403-11.

RESUMO Objetivo. Investigar os fatores associados à retenção de peso pós-parto segundo a cor da pele.

Métodos. Foi conduzido um estudo de coorte com quatro ondas de seguimento: aos 15 dias e 2, 6 e 9 meses pós-parto. As 450 participantes do estudo (idade entre 15 e 45 anos) foram recrutadas em uma maternidade e em um centro municipal de saúde localizados no Município do Rio de Janeiro, Brasil. A retenção de peso pós-parto foi definida como variável dependente. As co-variáveis investigadas foram idade da mãe, estado marital, habilidade para ler uma carta, hábito de fumar, renda familiar mensal em reais, número de filhos, idade ao primeiro parto e escore de atividade física no trabalho e no lazer. Os dados foram analisados em modelos de regressão linear longitudinal multivariados com efeitos mistos.

Resultados. Os fatores que permaneceram associados à retenção de peso pós-parto foram: para as mulheres brancas, tempo pós-parto $(\beta=-0,0061$, valor $\mathrm{P}=0,0002)$, estado marital (casada) solteira $\beta=-0,9279$, valor $\mathrm{P}=0,0457)$ e idade ao primeiro parto $(\beta=-0,1553$, valor $\mathrm{P}=$ $0,0364)$; para as pardas, tempo pós-parto $(\beta=-0,0062$, valor $\mathrm{P}<0,0001)$, estado marital (casada/solteira $\beta=-0,6000$, valor $\mathrm{P}=0,0145)$ e escore de atividade física no trabalho $(\beta=$ $-0,1068$, valor $\mathrm{P}=0,0003)$; e para as negras, hábito de fumar $(\beta=-1,4635$, valor $\mathrm{P}=0,0535)$ e escore de atividade física no lazer $(\beta=-0,2422$, valor $\mathrm{P}=0,0145)$.

Conclusões. Os fatores associados à retenção de peso pós-parto diferiram segundo a cor da pele. Entretanto, não se justifica a elaboração de intervenções específicas para cada categoria, especialmente porque, entre os fatores associados à retenção de peso, estão os escores para atividade física, que deve ser promovida em qualquer iniciativa para controlar a obesidade. Por outro lado, levando-se em conta as diferenças socioeconômicas entre as mulheres brancas, negras e pardas, novos estudos devem ser desenvolvidos para investigar mais a fundo o padrão de retenção de peso pós-parto segundo a cor da pele.

Palavras-chave Alterações do peso corporal, atividade física, cor de pele.

1 Universidade Federal do Rio de Janeiro (UFRJ), Instituto de Nutrição, Programa de Pós-Graduação em Nutrição (PPGN), Rio de Janeiro, RJ, Brasil.

2 UFRJ, Instituto de Nutrição, Departamento de $\mathrm{Nu}$ trição Social e Aplicada. Enviar correspondência a este autor no seguinte endereço: Universidade Federal do Rio de Janeiro, Instituto de Nutrição, Departamento de Nutrição Social e Aplicada, Avenida Brigadeiro Trompowsky s/n ${ }^{\circ}$, Bloco $\mathrm{J}, 2^{\circ}$ andar, sala 29, CEP 21941-590, Rio de Janeiro, RJ, Brasil. Fone: +55-21-2562-6595; fax: +55-21-22808243; e-mail: gkac@nutricao.ufrj.br
Diversos estudos recentes têm revelado que a obesidade é hoje um importante agravo nutricional, já consolidado, não sendo mais apenas um problema emergente de saúde pública (1-5). No Brasil, um exemplo recente consiste na Pesquisa sobre Orçamento Familiar (POF) realizada pelo Instituto Brasileiro de Geografia e Estatística
(IBGE) no período de 2002 a 2003. A POF revelou que $41 \%$ dos homens e $40 \%$ das mulheres com mais de 20 anos apresentaram algum grau de excesso de peso (indicado por um índice de massa corporal, ou IMC, $\geq 25,0 \mathrm{~kg} /$ $\mathrm{m}^{2}$ ) e que 9 e $13 \%$ dos homens e mulheres, respectivamente, eram obesos $\left(\mathrm{IMC} \geq 30,0 \mathrm{~kg} / \mathrm{m}^{2}\right)$. A gravidade do 
problema fica evidente ao serem consideradas as estimativas das prevalências de obesidade por faixa etária para as mulheres. Segundo a POF, a prevalência da obesidade para o Brasil como um todo variou de $4,7 \%$ em mulheres de 20 a 24 anos a $21,8 \%$ em mulheres de 55 a 64 anos (6).

Tendo em vista a elevada magnitude do sobrepeso e da obesidade, é importante implementar estratégias consistentes, como as recentemente recomendadas pela Organização Mundial da Saúde (OMS) (7). Segundo a OMS, o objetivo maior consiste em identificar em que momentos biológicos é possível prevenir o ganho de peso. Para as mulheres, o momento de maior risco de ganho de peso ocorre na idade reprodutiva, mais especificamente durante a gestação e nos 2 primeiros anos pós-parto (8-11). Os agravos nutricionais mais comuns nesse período são o ganho de peso gestacional excessivo e a retenção de peso pósparto (RPPP). No Brasil, ainda são escassos os estudos com mulheres em idade reprodutiva (12) e, mais especificamente, com mulheres no pós-parto (13-18).

Em geral, a RPPP é determinada por múltiplos fatores (9-11). Entre eles, destaca-se o elevado ganho de peso gestacional (17-20) e a baixa duração da amamentação $(14,16,21,22)$, sendo o efeito deste último ainda contraditório (23). Um aspecto particularmente pouco estudado em relação à RPPP consiste no efeito da variável cor da pele (1, 24-27). Considerando-se essa lacuna na literatura, o presente estudo apresenta dados sobre determinantes da RPPP para três categorias de cor da pele em mulheres do Município do Rio de Janeiro acompanhadas durante 9 meses após o parto.

\section{MATERIAIS E MÉTODOS}

\section{Delineamento, critérios de seleção e exclusão e perdas de seguimento}

Quatrocentas e cinqüenta mulheres foram recrutadas e acompanhadas durante 9 meses após o parto em um estudo longitudinal prospectivo. $\mathrm{O}$ estudo foi realizado no Centro Municipal de Saúde Marcolino Candau, localizado na região central do Município do Rio de Janeiro, Brasil, entre maio de 1999 e abril de 2001. Foram realizadas quatro ondas de seguimento: aos 15 dias e (aproximadamente) aos 2, 6 e 9 meses. Nessas ocasiões, foram coletados dados antropométricos e covariáveis. As mulheres foram recrutadas em três locais: na maternidade central da área, durante a rotina de prénatal e durante a rotina de vacinação com BCG; e em dois pontos no próprio Centro de Saúde Marcolino Candau. Os critérios de seleção foram ter entre 15 e 45 anos, ter menos de 30 dias de pós-parto na data da primeira entrevista, estar livre de doenças crônicas, ter tido o filho com idade gestacional superior a 35 semanas, não apresentar gestação gemelar e residir na área programática do estudo. Os critérios de exclusão foram não apresentar dados sobre o peso pré-gestacional $(n=13)$ e apresentar valores de retenção de peso pós-parto fora do intervalo de \pm 3 desvios padrão (DP), que correspondeu ao intervalo de $-10 \mathrm{~kg}$ a $16 \mathrm{~kg}$. Como esses limites cobrem aproximadamente $99 \%$ das observações da distribuição da variável de RPPP, acreditamos ter excluído as mulheres que potencialmente tenham informado o peso pré-gestacional com erro. O padrão de perdas foi avaliado levando-se em consideração a distribuição da taxa final de seguimento (número de mulheres com seguimento completo/número de mulheres que entraram na coorte) segundo diversas co-variáveis importantes, descritas mais adiante. As diferenças nas taxas finais de seguimento foram avaliadas segundo o teste do qui-quadrado $\left(\chi^{2}\right)$ para proporções. Detalhes adicionais sobre o delineamento, critérios de seleção e exclusão e padrão de perdas de seguimento podem ser obtidos em outras publicações $(15-18,28)$.

O presente projeto foi submetido e aprovado pela Comissão de Ética do Núcleo de Estudos de Saúde Coletiva (NESC) da Universidade Federal do
Rio de Janeiro (UFRJ) e está de acordo com os princípios éticos da não maleficência, beneficência, justiça e autonomia, contidos na Resolução 196/96 do Conselho Nacional de Saúde. Todas as participantes assinaram termo de consentimento, obtido de forma livre e espontânea, após terem sido feitos todos os esclarecimentos necessários.

\section{Variável dependente e co-variáveis}

A retenção de peso pós-parto foi definida como a variável dependente no presente estudo. O cálculo dessa variável levou em consideração a diferença entre o peso medido em cada onda de seguimento e o peso pré-gestacional informado pela mãe na primeira onda de seguimento. Essa variável foi trabalhada sob formato contínuo e apresentou distribuição normal.

A variável cor da pele da mãe foi trabalhada no estudo como variável de estratificação. As mulheres foram classificadas pelo pesquisador principal (GK) nas três categorias consideradas para fins de censo no Brasil, a saber: negras, pardas e brancas (29). O entrevistador recebeu treinamento prévio para a classificação das mães, com o objetivo de minimizar a ocorrência de viés de má classificação.

As co-variáveis listadas a seguir foram selecionadas de um amplo banco de dados para análise neste estudo, em função de sua importância na determinação da RPPP: idade da mãe sob o formato contínuo (anos) e categórico (15 a 19, 20 a 29 e 30 a 45 anos); estado marital (casada, em união ou solteira); habilidade para ler uma carta (sim, não); hábito de fumar (sim, não); renda familiar total em reais sob o formato contínuo e categórico $(<\mathrm{R} \$$ $480,00$ ou $\geq R \$ 480,00)$; paridade sob o formato discreto (número de filhos) e categórico (1, 2 e $\geq 3$ filhos); idade ao primeiro parto sob o formato contínuo (anos) e categórico ( $\leq 20$ e $>20$ anos); escore de atividade física no trabalho sob o formato contínuo e categórico e escore de atividade física no lazer sob o formato contínuo e categórico. 
Não são apresentados neste estudo resultados para co-variáveis que não mostraram associação com a retenção de peso no modelo final em qualquer uma das categorias de cor da pele, exceto renda familiar total (são apresentados apenas dados de freqüência) e idade da mãe, que foi incluída em todos os modelos. Para a variável de renda familiar total (mensal), optamos por usar o valor mediano para a classificação dos indivíduos em apenas dois grupos, uma vez que a divisão em quartis diluiria excessivamente a amostra. Em relação à variável idade ao primeiro parto, diversas categorias dicotômicas foram testadas com o objetivo de captar o seu efeito no desfecho, sendo a categorização aqui apresentada a que melhor desempenhou essa função. A criação dos escores de atividade física baseou-se na aplicação de um questionário validado, que compreende cinco questões referentes a atividade física no trabalho e seis questões referentes a atividade física no lazer. Cada opção de resposta equivale a pontos específicos e os escores são calculados somando-se todos os pontos. Quanto maior o valor do escore, maior o gasto energético (30). Os pontos de corte para os escores de atividade física foram definidos em função do valor mediano observado em cada onda de seguimento, a saber: atividade física no trabalho, visita 1 , mediana $=6$; visita 2 , mediana $=$ 7 ; visita 3 , mediana $=9$; e visita 4 , mediana $=8,5$; e atividade física no lazer, visita 1 , mediana $=1$; visitas 2,3 e 4 , mediana $=2$.

A variável de tempo pós-parto foi calculada levando-se em consideração a data de cada onda de seguimento e a data de nascimento da criança, em dias. Trata-se de uma variável de grande importância no desenvolvimento de todos os modelos longitudinais. É preciso mencionar ainda que algumas co-variáveis foram obtidas depois da primeira entrevista, ou seja, na segunda (habilidade para ler uma carta) e terceira (paridade e idade ao primeiro parto) ondas de seguimento. Dessa forma, o número de observações para essas co-variáveis é menor.

\section{Análise estatística}

A análise estatística foi desenvolvida em etapas e teve sempre, como variável de estratificação, a cor da pele da mãe, ou seja, todas as análises foram desenvolvidas separadamente para cada categoria de cor da pele. $\mathrm{Na}$ primeira etapa foram realizadas apenas análises descritivas. Inicialmente, apresentou-se a distribuição de freqüências para as co-variáveis selecionadas. As proporções foram comparadas segundo o teste do $\chi^{2}$. Na seqüência foram apresentados valores médios e intervalos de confiança de 95\% (IC95\%) para a retenção de peso pós-parto ao longo das quatro ondas de seguimento, segundo variáveis selecionadas.

A segunda etapa da análise envolveu o desenvolvimento de modelos bivariados de regressão linear longitudinal com efeitos mistos, levando-se em consideração apenas a retenção de peso pós-parto e cada uma das covariáveis selecionadas, controlando para o tempo pós-parto, em dias. As variáveis categóricas foram analisadas como fator (dummies), permitindo estimativas para cada um dos estratos em relação à categoria de referência. Essas análises geraram coeficientes de regressão bivariada específicos e valores de erro padrão (EP). Contudo, nos resultados do presente estudo, foi apresentada apenas a informação sobre o nível de significância para as co-variáveis estatisticamente associadas à RPPP. Essas análises nortearam o desenvolvimento do modelo multivariado de regressão longitudinal.

Na terceira e última etapa, foi feita uma modelagem longitudinal multivariada com o procedimento de efeitos mistos (linear mixed effects). Os modelos foram construídos levando-se em consideração a variável tempo pósparto, em dias, e as variáveis que apresentaram valores de $P<0,20$ na análise de regressão longitudinal bivariada. As variáveis que apresentaram valores de $P<0,10$ foram mantidas no modelo final com o objetivo de controlar o confundimento residual.
A avaliação da qualidade dos modelos foi feita levando-se em consideração critérios globais como os de Akaike e logLik (log likelihood). No que diz respeito à qualidade do ajuste, todos os modelos foram avaliados com base em gráficos de dispersão dos resíduos para verificar a presença de algum padrão específico; com base em gráficos quantil-quantil (q-q) para verificar a normalidade dos resíduos; e em gráficos com função de autocorrelação entre as medidas repetidas. Detalhes adicionais sobre o processo de modelagem com efeitos mistos podem ser obtidos nos artigos de Pinheiro e Bates (31) e Spyriades et al. (32).

\section{RESULTADOS}

Iniciaram o estudo de seguimento 169 mulheres brancas, 199 pardas e 82 negras, totalizando 450 mulheres. A tabela 1 apresenta a distribuição de freqüência para as variáveis selecionadas segundo a cor da pele. Observa-se que as mulheres pardas e negras foram classificadas com maior freqüência na faixa etária entre 15 e 19 anos (valor $P=0,0153$ ), e que as pardas e negras apresentaram idade ao primeiro parto $\leq 20$ anos com maior freqüência (valor $P=0,0104$ ).

As tabelas 2, 3 e 4 apresentam a evolução ( $x \pm \mathrm{IC} 95 \%)$ da RPPP segundo variáveis selecionadas para mulheres brancas, pardas e negras, respectivamente. Segundo a variável faixa etária, as mulheres brancas apresentaram os valores médios de RPPP mais elevados ao ínicio do estudo, seguidas das negras e pardas. Aos 9 meses pós-parto, as negras apresentaram os maiores valores para todas as faixas de idade (tabela 4). Em relação ao estado marital, observou-se que as solteiras pardas foram as únicas a retornar ao peso pré-gestacional aos 9 meses pós-parto, tendo experimentado a maior redução absoluta na retenção de peso pós-parto entre a primeira medida e o final do estudo $(-3,2 \mathrm{~kg})$ (tabela 3). As mulheres que tiveram o primeiro filho com $\leq 20$ anos apresentaram valores sistemati- 
TABELA 1. Distribuição de frequência das variáveis selecionadas segundo a cor da pele em 450 mulheres acompanhadas durante 9 meses pós-parto, Rio de Janeiro, Brasil, 1999 a 2001

\begin{tabular}{|c|c|c|c|c|c|c|}
\hline Variáveis & Branca & No. $\%$ & Parda & No. \% & Negra & No. $\%$ \\
\hline \multicolumn{7}{|l|}{ Faixa etária (anos) ${ }^{a}$} \\
\hline 15 a 19 & (31) & 18,3 & $(50)$ & 25,1 & (22) & 26,8 \\
\hline 20 a 29 & (90) & 53,3 & (120) & 60,3 & (41) & 50,0 \\
\hline 30 a 45 & (48) & 28,4 & (29) & 14,6 & (19) & 23,2 \\
\hline \multicolumn{7}{|l|}{ Estado marital } \\
\hline Casada & (35) & 20,7 & (28) & 14,1 & (13) & 15,9 \\
\hline Em união & (96) & 56,8 & (114) & 57,3 & (46) & 56,1 \\
\hline Solteira & (38) & 22,5 & $(57)$ & 28,6 & (23) & 28,0 \\
\hline \multicolumn{7}{|l|}{ Hábito de fumar } \\
\hline Sim & (18) & 10,7 & (37) & 18,6 & (14) & 17,1 \\
\hline Não & (151) & 89,3 & (162) & 81,4 & (68) & 82,9 \\
\hline \multicolumn{7}{|c|}{ Habilidade para ler uma carta } \\
\hline Sim & (131) & 86,8 & (149) & 85,1 & (56) & 82,4 \\
\hline Não & $(20)$ & 13,2 & (26) & 14,9 & (12) & 17,6 \\
\hline \multicolumn{7}{|c|}{ Renda familiar total (reais) ${ }^{\mathrm{b}}$} \\
\hline$<480$ & $(66)$ & 39,1 & (109) & 55,8 & $(46)$ & 56,1 \\
\hline$\geq 480$ & $(103)$ & 60,9 & $(90)$ & 45,2 & (36) & 43,9 \\
\hline \multicolumn{7}{|c|}{ Paridade (número de filhos) } \\
\hline Um & (53) & 42,4 & (64) & 45,1 & (28) & 50,9 \\
\hline Dois & $(45)$ & 36,0 & (42) & 29,6 & (9) & 16,4 \\
\hline Três ou mais & (27) & 21,6 & (36) & 25,4 & (18) & 32,7 \\
\hline \multicolumn{7}{|c|}{ Idade ao primeiro parto (anos) } \\
\hline$\leq 20$ & (60) & 48,0 & (93) & 65,5 & (35) & 63,6 \\
\hline$>20$ & (65) & 52,0 & (49) & 34,5 & (20) & 36,4 \\
\hline \multicolumn{7}{|c|}{ Escore de atividade física no trabalhoc } \\
\hline$<$ mediana & (78) & 46,4 & (82) & 41,2 & (38) & 46,3 \\
\hline > mediana & (90) & 53,6 & (117) & 58,8 & (44) & 53,7 \\
\hline \multicolumn{7}{|c|}{ Escore de atividade física no lazerc } \\
\hline$<$ mediana & (76) & 45,2 & (77) & 38,7 & (26) & 31,7 \\
\hline$>$ mediana & (92) & 54,8 & (122) & 61,3 & (56) & 68,3 \\
\hline
\end{tabular}

camente mais elevados de RPPP, independentemente da cor da pele.

A análise referente ao escore de atividade física no trabalho revelou que, entre as brancas, a RPPP foi maior nas mulheres classificadas com escore acima da mediana. Observou-se comportamento inverso para as mulheres pardas e negras. Em relação ao escore de atividade física no lazer, verificouse, entre as brancas e pardas, maiores valores de retenção entre as mulheres classificadas com escore acima da mediana, exceto aos 9 meses pós-parto para brancas. Já entre as negras, o comportamento foi inverso (tabelas 2, 3 e 4).

Resultados não apresentados da modelagem bivariada de regressão linear longitudinal revelaram que, do elenco de variáveis estudadas, o es- tado marital e a idade ao primeiro parto, para brancas; o estado marital e o escore de atividade física no trabalho, para pardas; o hábito de fumar e o escore de atividade física no lazer, para negras, apresentaram significância estatística $<0,20$ e foram consideradas como candidatas ao modelo final.

Os dados da tabela 5 revelam que distintas variáveis permaneceram significativamente associadas à retenção de peso pós-parto para cada categoria de cor da pele. No caso das mulheres brancas, essas variáveis foram tempo pós-parto (valor $P=0,0002$ ), estado marital (casada/solteira, valor $P=$ $0,0457)$ e idade ao primeiro parto (valor $P=0,0364$ ); para as pardas, tempo pós-parto (valor $P<0,0001$ ), estado marital (casada/solteira, valor $P=0,0145)$ e escore de atividade física no trabalho (valor $P=0,0003$ ); e para as negras, hábito de fumar (valor $P=$ 0,0535 ) e escore de atividade física no lazer (valor $P=0,0145$ ).

\section{DISCUSSÃO}

Os resultados do presente estudo revelaram que os fatores associados à RPPP diferiram entre as três categorias de cor da pele aqui estudadas. Diversos estudos nacionais e internacionais têm revelado que a obesidade materna se constitui em agravo mais freqüente nas mulheres negras do que nas brancas $(1,24-27)$. No presente estudo, os valores de RPPP também foram maio- 
TABELA 2. Evolução na retenção de peso em 169 mulheres brancas acompanhadas durante 9 meses pós-parto segundo variáveis selecionadas, Rio de Janeiro, Brasil, 1999 a 2001

\begin{tabular}{|c|c|c|c|c|c|c|c|c|c|c|c|c|}
\hline \multirow[b]{3}{*}{ Variáveis } & \multicolumn{12}{|c|}{ Retenção de peso pós-parto } \\
\hline & \multicolumn{3}{|c|}{0,5 mês } & \multicolumn{3}{|c|}{2 meses } & \multicolumn{3}{|c|}{6 meses } & \multicolumn{3}{|c|}{9 meses } \\
\hline & No. & $\begin{array}{l}\text { Média } \\
(\mathrm{kg})\end{array}$ & IC95\% & No. & $\begin{array}{l}\text { Média } \\
(\mathrm{kg})\end{array}$ & IC95\% & No. & $\begin{array}{c}\text { Média } \\
(\mathrm{kg})\end{array}$ & IC95\% & No. & $\begin{array}{c}\text { Média } \\
(\mathrm{kg})\end{array}$ & IC95\% \\
\hline \multicolumn{13}{|l|}{ Faixa etária (anos) } \\
\hline 15 a 19 & 31 & 6,4 & 4,9 a 7,9 & 28 & 5,1 & 3,7 a 6,5 & 23 & 3,8 & 1,8 a 5,8 & 19 & 2,6 & 0,1 a 5,0 \\
\hline 20 a 29 & 90 & 4,7 & 3,7 a 5,7 & 78 & 4,1 & 3,2 a 5,0 & 63 & 3,2 & 2,0 a 4,3 & 61 & 2,7 & 1,5 a 4,0 \\
\hline \multicolumn{13}{|l|}{ Estado marital ${ }^{\mathrm{a}}$} \\
\hline Casada & 35 & 6,6 & 5,0 a 8,1 & 31 & 5,1 & 3,4 a 6,7 & 25 & 4,2 & 2,3 a 6,0 & 22 & 3,2 & 1,0 a 5,4 \\
\hline União & 96 & 4,7 & 3,8 a 5,5 & 87 & 4,4 & 3,6 a 5,2 & 73 & 3,6 & 2,4 a 4,7 & 66 & 3,4 & 2,1 a 4,6 \\
\hline Solteira & 38 & 6,0 & 4,3 a 7,6 & 33 & 5,0 & 3,6 a 6,4 & 27 & 3,9 & 2,2 a 5,6 & 26 & 3,2 & 1,3 a 5,1 \\
\hline \multicolumn{13}{|l|}{ Hábito de fumar } \\
\hline Sim & 18 & 5,4 & 2,8 a 8,0 & 16 & 5,6 & 3,1 a 8,1 & 14 & 5,6 & 2,6 a 8,6 & 15 & 6,1 & 2,8 a 9,5 \\
\hline Não & 151 & 5,3 & 4,6 a 6,1 & 135 & 4,6 & 3,9 a 5,2 & 111 & 3,5 & 2,7 a 4,4 & 99 & 2,9 & 1,9 a 3,8 \\
\hline \multicolumn{13}{|c|}{ Habilidade para ler uma carta } \\
\hline \multicolumn{13}{|c|}{ Paridade (número de filhos) } \\
\hline Dois & 45 & 4,6 & 3,2 a 6,1 & 45 & 4,7 & 3,5 a 6,0 & 45 & 3,9 & 2,5 a 5,2 & 43 & 3,6 & 2,1 a 5,2 \\
\hline Três ou mais & 27 & 5,8 & 4,2 a 7,5 & 27 & 6,0 & 4,4 a 7,5 & 27 & 6,0 & 4,2 a 7,9 & 24 & 5,6 & 3,6 a 7,7 \\
\hline \multicolumn{13}{|c|}{$\begin{array}{l}\text { Idade ao primeiro parto } \\
(\text { anos) })^{\text {a }}\end{array}$} \\
\hline$\leq 20$ & 60 & 5,9 & 5,1 a 6,8 & 60 & 5,1 & 4,3 a 6,0 & 60 & 4,6 & 3,3 a 5,8 & 53 & 3,6 & 2,1 a 5,0 \\
\hline$>20$ & 65 & 4,5 & 3,3 a 5,6 & 65 & 4,0 & 3,1 a 5,0 & 65 & 3,0 & 1,9 a 4,1 & 61 & 3,0 & 1,8 a 4,3 \\
\hline \multicolumn{13}{|c|}{$\begin{array}{l}\text { Escore de atividade física no } \\
\text { trabalhob }\end{array}$} \\
\hline$<$ mediana & 78 & 5,1 & 4,1 a 6,2 & 57 & 3,9 & 3,0 a 4,8 & 56 & 3,4 & 2,1 a 4,6 & 49 & 3,6 & 2,1 a 5,0 \\
\hline$>$ mediana & 90 & 5,5 & 4,6 a 6,5 & 94 & 5,1 & 4,3 a 6,0 & 69 & 4,1 & 3,0 a 5,2 & 65 & 3,1 & 1,8 a 4,3 \\
\hline \multicolumn{13}{|c|}{$\begin{array}{l}\text { Escore de atividade física no } \\
\text { lazerb }\end{array}$} \\
\hline$<$ mediana & 76 & 5,8 & 4,8 a 6,8 & 34 & 4,3 & 2,9 a 5,7 & 26 & 2,8 & 1,0 a 4,6 & 27 & 2,2 & 0,4 a 3,9 \\
\hline
\end{tabular}

a Variáveis associadas com a retenção de peso pós-parto na análise bivariada de regressão longitudinal linear com efeitos mistos controlando para tempo pós-parto, em dias.

Escore de atividade física baseado na escala de Shapiro et al. (29). Para atividade física no trabalho, a mediana na visita 1 foi 6 ; visita 2, mediana $=7$; visita 3, mediana = 9; e visita 4, mediana $=8,5$. Para atividade física, no lazer a mediana na visita 1 foi 1 ; visitas 2,3 e 4 , mediana $=2$.

res em negras quando comparadas com pardas e brancas. Considerandose esse histórico, é importante que, em populações com diversidade étnica, a exemplo do Brasil, a categoria cor da pele seja considerada em análises sobre o padrão de distribuição e determinação da RPPP.

Observou-se, no presente estudo, que o estado marital esteve associado à RPPP para as mulheres brancas $\mathrm{e}$ pardas. Entre as negras não se observou nenhum efeito dessa variável. Em geral, as mulheres solteiras apresentaram os menores valores de retenção de peso ao final do estudo. As mulheres solteiras pardas, por exemplo, foram as únicas a praticamente retornar ao peso pré-gestacional aos 9 meses pósparto. São ainda escassos os estudos enfocando o estado marital como determinante da RPPP, e os estudos revisados não são conclusivos. Janney et al. (22), por exemplo, observaram que as mulheres não casadas apresentaram maior risco de desenvolver obesidade, resultado conflitante com os dados aqui apresentados. Por outro lado, Gunderson et al. (33) não observaram associação entre o estado marital e a obesidade materna, enquanto Wolfe et al. (34), descreveram um maior acúmulo de peso associado à paridade em mulheres não casadas entre 25 e 45 anos, mesmo depois de controlado o efeito de variáveis de confusão.
A idade ao primeiro parto, a habilidade para ler uma carta e a paridade também permaneceram no modelo final de regressão longitudinal para as mulheres brancas, as duas últimas com significância limítrofe. Verificou-se uma relação inversa entre idade ao primeiro parto e retenção de peso, ou seja, quanto menor a idade ao primeiro parto, maior a retenção de peso. Gunderson et al. (33) também observaram maiores valores de IMC em mulheres com idade precoce de gravidez. O efeito da variável idade ao primeiro parto já havia sido identificado em análises anteriores sem estratificação por cor da pele, onde observou-se que as mulheres com idade precoce na pri- 
TABELA 3. Evolução na retenção de peso em 199 mulheres pardas acompanhadas durante 9 meses pós-parto segundo variáveis selecionadas, Rio de Janeiro, Brasil, 1999 a 2001

\begin{tabular}{|c|c|c|c|c|c|c|c|c|c|c|c|c|}
\hline \multirow[b]{3}{*}{ Variáveis } & \multicolumn{12}{|c|}{ Retenção de peso pós-parto } \\
\hline & \multicolumn{3}{|c|}{0,5 mês } & \multicolumn{3}{|c|}{2 meses } & \multicolumn{3}{|c|}{6 meses } & \multicolumn{3}{|c|}{9 meses } \\
\hline & No. & $\begin{array}{l}\text { Média } \\
(\mathrm{kg})\end{array}$ & IC95\% & No. & $\begin{array}{l}\text { Média } \\
(\mathrm{kg})\end{array}$ & IC95\% & No. & $\begin{array}{c}\text { Média } \\
(\mathrm{kg})\end{array}$ & IC95\% & No. & $\begin{array}{l}\text { Média } \\
(\mathrm{kg})\end{array}$ & IC95\% \\
\hline \multicolumn{13}{|l|}{ Faixa etária (anos) } \\
\hline 15 a 19 & 50 & 3,8 & 2,5 a 5,1 & 41 & 3,1 & 1,6 a 4,6 & 33 & 1,5 & $-0,1$ a 3,2 & 27 & 1,2 & $-0,6$ a 3,0 \\
\hline 20 a 29 & 120 & 4,6 & 3,7 a 5,4 & 104 & 3,8 & 2,9 a 4,8 & 84 & 3,1 & 2,1 a 4,2 & 78 & 2,6 & 1,5 a 3,7 \\
\hline Casada & 28 & 6,2 & 4,3 a 8,1 & 29 & 5,3 & 3,2 a 7,4 & 26 & 4,5 & 2,6 a 6,4 & 26 & 3,7 & 1,9 a 5,5 \\
\hline União & 114 & 4,4 & 3,6 a 5,2 & 101 & 3,9 & 3,0 a 4,7 & 80 & 3,1 & 2,2 a 4,1 & 69 & 2,8 & 1,8 a 3,8 \\
\hline Solteira & 57 & 3,4 & 2,2 a 4,6 & 45 & 2,0 & 0,8 a 3,3 & 36 & 0,5 & $-1,0$ a 2,1 & 33 & 0,2 & $-1,4$ a 1,9 \\
\hline \multicolumn{13}{|l|}{ Hábito de fumar } \\
\hline Sim & 37 & 4,6 & 3,0 a 6,1 & 33 & 3,3 & 1,7 a 4,8 & 26 & 2,4 & 0,8 a 4,1 & 23 & 2,3 & 0,4 a 4,3 \\
\hline Não & 162 & 4,3 & 3,6 a 5,0 & 142 & 3,7 & 2,9 a 4,5 & 116 & 2,8 & 1,9 a 3,7 & 105 & 2,3 & 1,4 a 3,2 \\
\hline \multicolumn{13}{|c|}{ Habilidade para ler uma carta } \\
\hline Dois & 42 & 4,1 & 2,7 a 5,6 & 42 & 4,3 & 2,8 a 5,8 & 42 & 3,3 & 2,0 a 4,7 & 37 & 3,1 & 1,6 a 4,6 \\
\hline Três ou mais & 36 & 4,9 & 3,6 a 6,2 & 36 & 3,9 & 2,6 a 5,3 & 36 & 3,6 & 2,0 a 5,1 & 35 & 3,4 & 1,9 a 4,9 \\
\hline \multicolumn{13}{|c|}{$\begin{array}{l}\text { Idade ao primeiro parto } \\
\text { (anos) }\end{array}$} \\
\hline$\leq 20$ & 93 & 4,4 & 3,7 a 5,2 & 93 & 3,8 & 3,0 a 4,7 & 93 & 2,9 & 1,9 a 3,8 & 85 & 2,6 & 1,6 a 3,6 \\
\hline$>20$ & 49 & 4,2 & 3,0 a 5,2 & 49 & 3,1 & 2,0 a 4,2 & 49 & 2,4 & 1,1 a 3,8 & 43 & 1,7 & 0,4 a 3,1 \\
\hline \multicolumn{13}{|c|}{$\begin{array}{l}\text { Escore de atividade física no } \\
\text { trabalho }{ }^{a, b}\end{array}$} \\
\hline$<$ mediana & 82 & 4,7 & 3,6 a 5,7 & 62 & 4,5 & 3,2 a 5,7 & 76 & 3,1 & 2,1 a 4,2 & 78 & 2,1 & 1,1 a 3,1 \\
\hline$>$ mediana & 117 & 4,1 & 3,3 a 4,9 & 113 & 3,2 & 2,3 a 4,0 & 66 & 2,3 & 1,1 a 3,8 & 50 & 2,7 & 1,3 a 4,0 \\
\hline \multicolumn{13}{|c|}{$\begin{array}{l}\text { Escore de atividade física no } \\
\text { lazer }^{b}\end{array}$} \\
\hline$<$ mediana & 77 & 4,0 & 2,9 a 5,0 & 20 & 1,5 & $-0,5$ a 3,4 & 16 & 1,5 & $-1,0$ a 4,0 & 8 & 0,1 & $-3,1$ a 3,3 \\
\hline
\end{tabular}

a Variáveis associadas com a retenção de peso pós-parto na análise bivariada de regressão longitudinal linear com efeitos mistos controlando para tempo pós-parto, em dias.

${ }^{b}$ Escore de atividade física baseado na escala de Shapiro et al. (29). Para atividade física no trabalho, a mediana na visita 1 foi 6; visita 2, mediana = 7; visita 3, mediana = 9; e visita 4, mediana $=8,5$. Para atividade física no lazer, a mediana na visita 1 foi 1 ; visitas 2,3 e 4 , mediana $=2$.

meira gravidez apresentaram 2,80 vezes mais chance de reter $7,5 \mathrm{~kg}$ ou mais 9 meses após o parto (18). A presente análise apenas confirmou que o efeito dessa variável só ocorreu em mulheres brancas. $\mathrm{O}$ início precoce do ciclo reprodutivo, assim como a potencial ativação precoce de alguns hormônios relacionados com a obesidade, poderiam explicar o efeito da idade ao primeiro parto na RPPP (35). Em relação à paridade, observou-se que, mesmo o coeficiente não tendo apresentando valor significativo, a direção positiva do mesmo apontou para maior retenção entre mulheres com maior paridade. Esse comportamento, apesar de não ser totalmente concordante com os resultados descritos literatura, tem sido observado em alguns estudos (36).

Apesar da não significância estatística, foi interessante observar que as mulheres que não conseguiam ler uma carta apresentaram valores de retenção mais elevados em até $2 \mathrm{~kg}$, segundo o modelo final de regressão longitudinal, quando comparadas às mulheres que conseguiam ler uma carta. Considerando-se essa variável como proxy da variável escolaridade, observou-se que esse resultado está de acordo com pesquisas recentes, que verificaram que as mulheres de baixa escolaridade estão mais sujeitas a desenvolver obesidade (37). Análises anteriores baseadas em dados dessa mesma coorte revelaram que as mulheres com incapacidade ou dificuldade para ler adequadamente uma carta apresentaram 2,10 vezes mais chance de reter $\geq 7,5 \mathrm{~kg}$ ao final de 9 meses pós-parto (18).

Foi interessante observar que os escores que medem a atividade física apresentaram associação com a RPPP em mulheres pardas e negras, mas não entre as brancas. É ainda curioso que, entre as pardas, a associação tenha ocorrido com o escore para atividade física no trabalho, e, entre as negras, com o escore para atividade física no lazer. Esses resultados são conflitantes com o que relataram Schauberger et al. (38), que acompanharam 739 mulheres e concluí- 
TABELA 4. Evolução na retenção de peso em 82 mulheres negras acompanhadas durante 9 meses pós-parto segundo variáveis selecionadas, Rio de Janeiro, Brasil, 1999 a 2001

\begin{tabular}{|c|c|c|c|c|c|c|c|c|c|c|c|c|}
\hline \multirow[b]{3}{*}{ Variáveis } & \multicolumn{12}{|c|}{ Retenção de peso pós-parto } \\
\hline & \multicolumn{3}{|c|}{0,5 mês } & \multicolumn{3}{|c|}{2 meses } & \multicolumn{3}{|c|}{6 meses } & \multicolumn{3}{|c|}{9 meses } \\
\hline & No. & $\begin{array}{l}\text { Média } \\
(\mathrm{kg})\end{array}$ & IC95\% & No. & $\begin{array}{c}\text { Média } \\
(\mathrm{kg})\end{array}$ & IC95\% & No. & $\begin{array}{c}\text { Média } \\
(\mathrm{kg})\end{array}$ & IC95\% & No. & $\begin{array}{l}\text { Média } \\
(\mathrm{kg})\end{array}$ & IC95\% \\
\hline \multicolumn{13}{|l|}{ Faixa etária (anos) } \\
\hline 15 a 19 & 22 & 4,8 & 2,7 a 6,9 & 17 & 4,6 & 2,4 a 6,7 & 14 & 5,4 & 2,8 a 8,0 & 12 & 4,5 & 1,4 a 7,5 \\
\hline 20 a 29 & 41 & 4,3 & 2,8 a 5,8 & 34 & 3,7 & 2,0 a 5,5 & 28 & 3,0 & 1,0 a 4,9 & 26 & 3,2 & 1,0 a 5,4 \\
\hline Casada & 13 & 4,1 & 0,6 a 7,6 & 12 & 4,2 & 0,8 a 7,5 & 11 & 3,9 & 0,1 a 7,7 & 9 & 4,5 & $-0,1$ a 9,0 \\
\hline União & 46 & 4,7 & 3,4 a 5,9 & 37 & 4,1 & 2,7 a 5,5 & 30 & 4,7 & 2,8 a 6,7 & 29 & 4,7 & 2,7 a 6,7 \\
\hline Solteira & 23 & 5,2 & 3,2 a 7,2 & 19 & 4,5 & 2,2 a 6,7 & 14 & 3,5 & 1,4 a 5,6 & 12 & 2,9 & 0,1 a 5,7 \\
\hline \multicolumn{13}{|l|}{ Hábito de fumara } \\
\hline Sim & 14 & 6,6 & 3,8 a 9,5 & 10 & 6,9 & 3,7 a 10,0 & 7 & 6,7 & 2,1 a 11,2 & 6 & 4,5 & $-0,0$ a 9,0 \\
\hline Não & 68 & 4,3 & 3,3 a 5,4 & 58 & 3,8 & 2,6 a 4,9 & 48 & 3,9 & 2,5 a 5,3 & 44 & 4,2 & 2,6 a 5,8 \\
\hline \multicolumn{13}{|c|}{ Habilidade para ler uma carta } \\
\hline Dois & 9 & 5,3 & 1,5 a 9,2 & 9 & 4,0 & 0,5 a 7,5 & 9 & 2,6 & 0,6 a 4,6 & 8 & 2,5 & $-0,3$ a 5,3 \\
\hline Três ou mais & 18 & 5,9 & 3,6 a 8,2 & 18 & 5,3 & 3,0 a 7,6 & 18 & 5,3 & 2,3 a 8,3 & 16 & 5,5 & 2,0 a 8,9 \\
\hline \multicolumn{13}{|c|}{$\begin{array}{l}\text { Idade ao primeiro parto } \\
\text { (anos) }\end{array}$} \\
\hline$\leq 20$ & 35 & 4,7 & 3,5 a 6,0 & 35 & 4,3 & 3,0 a 5,7 & 35 & 5,0 & 3,2 a 6,8 & 32 & 4,9 & 2,9 a 6,9 \\
\hline$>20$ & 20 & 4,7 & 2,9 a 6,5 & 20 & 3,9 & 1,9 a 6,0 & 20 & 2,9 & 0,9 a 4,9 & 18 & 3,0 & 0,8 a 5,2 \\
\hline \multicolumn{13}{|c|}{$\begin{array}{l}\text { Escore de atividade física no } \\
\text { trabalhob }\end{array}$} \\
\hline$<$ mediana & 38 & 6,0 & 4,8 a 7,3 & 26 & 4,2 & 2,3 a 6,1 & 28 & 5,3 & 3,2 a 7,4 & 19 & 5,5 & 2,9 a 8,1 \\
\hline$>$ mediana & 44 & 3,6 & 2,1 a 5,1 & 42 & 4,3 & 2,9 a 5,6 & 27 & 3,1 & 1,5 a 4,8 & 31 & 3,4 & 1,6 a 5,2 \\
\hline \multicolumn{13}{|c|}{$\begin{array}{l}\text { Escore de atividade física no } \\
\text { lazera,b }\end{array}$} \\
\hline$<$ mediana & 26 & 5,0 & 3,2 a 6,7 & 11 & 5,2 & 2,2 a 8,1 & 5 & 4,7 & $-3,7$ a 13,2 & 9 & 3,9 & $-0,04$ a 7,9 \\
\hline
\end{tabular}

a Variáveis associadas com a retenção de peso pós-parto na análise bivariada de regressão longitudinal linear com efeitos mistos controlando para tempo pós-parto, em dias.

Escore de atividade física baseado na escala de Shapiro et al. (29). Para atividade física no trabalho, a mediana na visita 1 foi 6; visita 2, mediana $=7$; visita 3, mediana = 9; e visita 4, mediana $=8,5$. Para atividade física no lazer, a mediana na visita 1 foi 1 ; visitas 2,3 e 4 , mediana $=2$.

ram que a atividade física não influenciou nem o ganho de peso nem a RPPP.

Em relação à variável hábito de fumar, Pomerleau et al. (39), em um estudo realizado em Michigan, nos Estados Unidos, verificaram que, para as mulheres fumantes, o grande problema era lidar com o ganho de peso excessivo concomitantemente com os efeitos da abstinência da nicotina. Mesmo assim, os autores observaram que essas mulheres tiveram uma perda de peso pós-parto mais veloz do que as não fumantes.

Algumas questões metodológicas merecem ser discutidas. A primeira diz respeito ao uso da variável cor da pele, que foi medida pelo observador, e não pelos participantes do estudo, como querem alguns autores (40). Entretanto, não há evidências de viés de má classificação. Contribui para esse entendimento a interpretação de resultados não apresentados, que revelaram um padrão socioeconômico e demográfico compatível com aquele da população geral (41), ou seja, mais favorável para as mulheres brancas e menos favorável para as negras. Uma outra questão importante é o tratamento dispensado às potenciais perdas de seguimento, problema comum em estudos de coorte. Como já foi demonstrado em outras análises para o estudo de coorte como um todo (1518), não se observou um padrão de perda seletiva, nem mesmo quando as análises foram estratificadas pela cor da pele. Por outro lado, é importante destacar que um ponto positivo do presente estudo consistiu no uso de um procedimento robusto de análise, como é o caso dos modelos de efeitos mistos. Com o uso dessa abordagem analítica, a precisão e o poder para detectar diferenças estatisticamente significativas é maior do que nos estudos transversais e do que com a análise de variância (ANOVA) para medidas repetidas (31).

Em síntese, conclui-se que, mesmo sendo diferentes os determinantes da RPPP segundo a cor da pele, não existe evidência que indique a necessidade 
TABELA 5. Modelo final de regressão longitudinal para retenção de peso pós-parto segundo a cor da pele em mulheres acompanhadas durante 9 meses pós-parto, Rio de Janeiro, Brasil, 1999 a 2001 ${ }^{\text {a }}$

\begin{tabular}{lrrr}
\hline \multicolumn{1}{c}{ Variáveis } & $\begin{array}{c}\text { Coeficiente de } \\
\text { regressão }\end{array}$ & $\begin{array}{c}\text { Erro } \\
\text { padrão }\end{array}$ & Valor $P$ \\
\hline Brancas $^{\text {b }}$ & & & \\
Tempo pós-parto (dias) & $-0,0061$ & 0,0016 & 0,0002 \\
Estado marital (casada/solteira) & $-0,9279$ & 0,4597 & 0,0457 \\
Estado marital (casada/em união) & $-0,0747$ & 0,3013 & 0,8045 \\
Habilidade para ler uma carta (sim, não) & 2,0410 & 1,1379 & 0,0753 \\
Idade ao primeiro parto (anos) & $-0,1553$ & 0,0734 & 0,0364 \\
Paridade (número de filhos) & 0,6515 & 0,3768 & 0,0863 \\
Intercepto & 5,3910 & 2,1259 & 0,0116 \\
Critérios de Akaike & $2.377,5$ & & \\
Log likelihood & $-1.177,7$ & & \\
Pardas & & & \\
Tempo pós-parto (dias) & $-0,0062$ & 0,0016 & $<0,0001$ \\
Estado marital (casada/solteira) & $-0,6000$ & 0,2432 & 0,0145 \\
Estado marital (casada/em união) & $-0,5339$ & 0,4391 & 0,2255 \\
Escore de atividade física no trabalho & $-0,1068$ & 0,0289 & 0,0003 \\
Intercepto & 3,4884 & 0,7836 & $<0,0001$ \\
Critérios de Akaike & $2.377,3$ & & \\
Log likelihood & $-1.181,6$ & & \\
Negras & & & \\
Tempo pós-parto (dias) & $-0,0012$ & 0,0024 & 0,6282 \\
Hábito de fumar (sim/não) & $-1,4635$ & 0,7444 & 0,0535 \\
Escore de atividade física no lazer & $-0,2422$ & 0,0981 & 0,0145 \\
Intercepto & 3,4884 & 0,7836 & $<0,0001$ \\
Critérios de Akaike & $2.377,3$ & & \\
Log likelihood & $-1.181,6$ & & \\
\hline
\end{tabular}

a Todos os modelos foram ajustados para o efeito da variável de idade em anos.

b 494 observações em 131 mulheres.

${ }^{c} 644$ observações em 203 mulheres.

d 242 observações em 69 mulheres. de propostas específicas de intervenção, sobretudo porque, entre os determinantes que se diferenciam, estão incluídos os escores para atividade física no lazer e no trabalho, e toda e qualquer proposta de intervenção para a obesidade deve envolver a promoção do aumento da atividade física. É importante, entretanto, que novos estudos sejam desenvolvidos com o objetivo de investigar de forma mais consolidada a presença ou não de diferenças no padrão de distribuição da RPPP segundo a cor da pele, com base nas ainda intensas diferenças nos indicadores socioeconômicos.

Agradecimentos. Gilberto Kac recebeu bolsa de doutorado da Coordenação de Aperfeiçoamento de Pessoal de Nível Superior (CAPES) de 1999 a 2002. O projeto de pesquisa original foi financiado pela Fundação Universitária José Bonifácio, da Universidade Federal do Rio de Janeiro (FUJB/UFRJ), e pela Fundação de Amparo à Pesquisa do Estado do Rio de Janeiro (FAPERJ). Gilberto Kac é bolsista de produtividade do Conselho Nacional de Desenvolvimento Científico e Tecnológico (CNPq).

\section{REFERÊNCIAS}

1. Hidalgo CAG, Kac G, Velásquez-Meléndez G, Valente JG. Factors associated with overweight in Brazilian childbearing-age women according to skin color. Nutr Res. 2002;22(7): 785-94.

2. Wang Y, Monteiro C, Popkin B. Trends of obesity and underweight in older children and adolescents in the United States, Brazil, China and Russia. Am J Clin Nutr. 2002;75(6):971-7.

3. Batista Filho M, Rissin A. A transição nutricional no Brasil: tendências regionais e temporais. Cad Saude Publica. 2003;19(supl. 1): 181-91.

4. Kac G, Velásquez-Meléndez G. A transição nutricional e a epidemiologia da obesidade na América Latina. Cad Saude Publica. 2003;19 (supl. 1):s4-5.

5. Velásquez-Meléndez G, Pimenta AM, Kac G. Epidemiologia do sobrepeso e da obesidade e seus fatores determinantes em Belo Horizonte (MG), Brasil: estudo transversal de base populacional. Rev Panam Salud Publica. 2004; 16(5):308-14.

6. Instituto Brasileiro de Geografia e Estatística. Pesquisa de orçamentos familiares 20022003. Análise da disponibilidade domiciliar de alimentos e do estado nutricional no Brasil, 2004. Rio de Janeiro: IBGE; 2004. Disponível em http://www.ibge.gov.br/home/ estatistica/populacao/condicaodevida/pof/ 2002analise/pof2002analise.pdf. Acessado em setembro de 2005.

7. World Health Organization. Obesity: Preventing and managing the global epidemicReport of a WHO consultation on obesity. Genebra: WHO; 1998.

8. Ohlin A, Rossner S. Maternal body weight development after pregnancy. Int J Obes. 1990; 14(2):159-73.

9. Lederman SA. The effect of pregnancy weight gain on later obesity. Obstet Gynecol. 1993; 82(1):148-55.

10. Kac G. Fatores determinantes da retenção de peso no pós-parto: uma revisão da literatura. Cad Saude Publica. 2001;17(3):455-66.

11. Linné $Y$, Rossner $S$. Interrelationships between weight development and weight retention in subsequent pregnancies: the SPAWN study. Acta Obstet Gynecol Scand. 2003;82(4): 318-25.

12. Nucci LB, Duncan BB, Mengue SS, Branchtein L, Schmidt MI, Fleck ET. Assessment of weight gain during pregnancy in general prenatal care services in Brazil. Cad Saude Publica. 2001;17(6):1367-74.

13. Fórnes NS, Dorea JG. Subcutaneous fat changes in low-income lactating mothers and growth of breast-fed infants. J Am Coll Nutr. 1995;14(1):61-5.

14. Gigante DP, Victora CG, Barros FC. Breastfeeding has a limited long-term effect on anthropometry and body composition of Brazilian mothers. J Nutr. 2001;131(1):78-84.

15. Kac G, Benício MHDA, Velásquez-Meléndez G, Valente JG. Postpartum weight retention among women in Rio de Janeiro: a follow-up study. Cad Saude Publica. 2003;19(Supl. 1): 149S-61S.

16. Kac G, Benício MHDA, Velásquez-Meléndez G, Valente JG, Struchiner CJ. Breastfeeding and postpartum weight retention in a cohort of Brazilian women. Am J Clin Nutr. 2004; 79(3):487-93.

17. Kac G, Benício MHDA, Velásquez-Meléndez G, Valente JG, Struchiner CJ. Gestational weight gain and pre-pregnancy weight influence postpartum weight retention in a cohort of Brazilian women. J Nutr. 2004;134(3): 661-6.

18. Kac G, Benício MHDA, Velásquez-Meléndez G, Valente JG. Nine months postpartum weight retention predictors for Brazilian women. Public Health Nutr. 2004;7(5):661-8.

19. Keppel KG, Taffel SM. Pregnancy-related weight gain and retention: implications of the 
1990 Institute of Medicine Guidelines. Am J Publ Health. 1993;83(8):1100-3.

20. Abrams B, Selvin, S, Gunderson EP. Pregnancy weight gain: still controversial. Am. J Clin Nutr. 2000;71(Suppl. 1):1233S-41S.

21. Dewey KG, Heining MJ, Nommsen LA. Maternal weight-loss patterns during prolonged lactation. Am J Clin Nutr. 1993;58(2):162-6.

22. Janney CA, Zhang D, Sowers M. Lactation and weight retention. Am J Clin Nutr. 1997; 66(5):1116-24.

23. Butte NF, Hopkinson JM. Body composition changes during lactation are highly variable among women. J Nutr. 1998;128(2):381S-5S.

24. Kahan HS, Williamson DF, Stevens JA. Race and weight change in US women: the roles of socioeconomic and marital status. Am J Public Health. 1991;81(3):319-23.

25. Parker JD, Abrams B. Differences in postpartum weight retention between black and white mothers. Obstet Gynecol. 1993;81(5 Pt 1):768-74.

26. Boardley DJ, Sargent RG, Coker AL, Hussey JR, Sharpe PA. The relationship between diet, activity and other factors, and postpartum weight change by race. Obstet Gynecol. 1995; 86(5):834-8.

27. Caulfield LE, Witter FR, Stoltzfus RJ. Determinants of gestational weight gain outside the recommended ranges among black and white women. Obstet Gynecol. 1996;87(5 Pt 1): 760-6.

28. Kac G. Fatores determinantes da retenção de peso pós-parto em uma coorte de mulheres com nove meses de seguimento [tese de doutorado]. São Paulo: Faculdade de Saúde Pública da Universidade de São Paulo; 2002.

29. Instituto Brasileiro de Geografia e Estatística. Censo Demográfico 2000: características da população e dos domicílios. Rio de Janeiro: Instituto Brasileiro de Geografia e Estatística; 2000.

30. Shapiro S, Weinblat E, Frank CW, Sager RV. The HIP study of incidence and prognosis of coronary heart disease. J Chron Dis. 1965;18: 527-58.

31. Pinheiro JC, Bates DM. Mixed-effects models in S and S-PLUS. New York: Springer-Verlag; 2000.

32. Spyrides MHC, Struchiner CJ, Barbosa MTS, Kac G. Práticas de amamentação e outros determinantes do crescimento infantil nos primeiros meses de vida, Rio de Janeiro, 19992001. Cad Saude Publica. 2005;21(3):756-66

33. Gunderson EP, Abrams B, Selvin S. The relative importance of gestational gain and maternal characteristics associated with the risk of becoming overweight after pregnancy. Int J Obes. 2000;24(12):1660-8

34. Wolfe WS, Sobal J, Olson CM, Frongillo EA Jr, Williamson DF. Parity-associated weight gain and its modification by sociodemographic and behavioral factors: a prospective analysis in US women. Int J Obes Relat Metab Disord. 1997;21(9):802-10.

35. Kac G, Velásquez-Meléndez G, Valente JG. Menarca, gravidez precoce e obesidade em mulheres brasileiras selecionadas em um centro de saúde de Belo Horizonte, Minas Gerais, Brasil. Cad Saude Publica. 2003;19(Supl. 1): s111-8.

36. Björkelund C, Lissner L, Andersson S, Lapidus L, Bengtsson $\mathrm{C}$. Reproductive history in relation to relative weight and fat distribution. Int J Obes. 1996;20(3):213-9.

37. Monteiro CA, Conde WL. A tendência secular da obesidade segundo estratos sociais: Nordeste e Sudeste do Brasil, 1975-1989-1997. Arq Bras Endocrinol Metab. 1999;43(3):186-94.

38. Schauberger CH, Rooney BL, Brimer LM. Factors that influence weight loss in the puerperium. Obstet Gynecol. 1992;79(3):424-9.

39. Pomerleau CS, Brouwer RJ, Jones LT, Weight concern in women smokers during pregnancy and postpartum. Addict Behav. 2000;25(5): 759-67.

40. Maio MC, Santos RV. Políticas de cotas raciais, os "olhos da sociedade" e os usos da antropologia: $\mathrm{O}$ caso do vestibular da Universidade de Brasília (UnB). Horiz Antropol. 2005; 11(23):181-214

41. Leal MC, Gama SGN, Cunha CB. Racial, sociodemographic, and prenatal and childbirth care inequalities in Brazil, 1999-2001. Rev Saude Publica. 2005;39(1):100-7.

Manuscrito recebido em 17 de fevereiro de 2005. Aceito em versão revisada em 12 de agosto de 2005.

ABSTRACT Objective. To investigate factors associated with postpartum weight retention according to skin color in the city of Rio de Janeiro, Brazil, as part of the effort to help control overweight and obesity.

Determinants of postpartum weight retention according to skin color among women in Rio de Janeiro, Brazil

Methods. We carried out a cohort study with data collection at four points after delivery: 15 days, 2 months, 6 months, and 9 months. The 450 participants (15 to 45 years of age) were recruited from a maternity center and a city health center in Rio de Janeiro. Postpartum weight retention was defined as the dependent variable. The following covariates were investigated: maternal age, marital status, ability to read a letter, smoking, monthly family income, number of children, age at first delivery, a score for physical activity at work, and a score for physical activity during leisure time. The data were analyzed using multivariate linear mixed-effects models for longitudinal data.

Results. The factors that remained associated with postpartum weight retention for white women were time after delivery $(\beta=-0.0061, P=0.0002)$, marital status (married/single $\beta=-0.9279, P=0.0457)$, and age at first delivery $(\beta=-0.1553, P=$ $0.0364)$. The factors for brown women were time after delivery $(\beta=-0.0062, P<$ 0.0001 ), marital status (married/single $\beta=-0.6000, P=0.0145$ ), and work physical activity score $(\beta=-0.1068, P=0.0003)$. The factors for black women were smoking $(\beta=$ $-1.4635, P=0.0535)$ and score for physical activity during leisure time $(\beta=-0.2422$, $P=0.0145$ )

Conclusions. The factors associated with postpartum weight retention were different for the three skin-color categories. Nevertheless, the data do not indicate the need for different interventions for the three skin-color groups. That is particularly true because the physical activity scores were among the variables associated with weight retention, and any initiative to control obesity should take physical activity into consideration. On the other hand, given the socioeconomic differences among the three skin-color categories, new studies should be developed to investigate in more detail the pattern of postpartum weight retention according to skin color.

Key words Body weight, weight gain, obesity, pregnancy, skin pigmentation, motor activity, Brazil. 